\title{
Electromagnetic Corrections to Meson Masses and the HVP
}

\author{
Vera Gülpers;, James Harrison', Andreas Jüttner, Christopher Sachrajda \\ School of Physics and Astronomy, University of Southampton \\ Southampton SO17 1BJ, United Kingdom \\ E-mail: \{V.M.Guelpers, J.Harrison\} esoton.ac.uk
}

Peter Boyle, Antonin Portelli

School of Physics and Astronomy, University of Edinburgh

Edinburgh EH9 3JZ, United Kingdom

\section{RBC and UKQCD Collaborations}

We present an exploratory study of the electromagnetic corrections to meson masses and the hadronic vacuum polarization using $N_{f}=2+1$ Domain Wall fermions. These corrections are estimated with two different approaches, a stochastic approach using $U(1)$ gauge configurations for the photon fields, and a perturbative approach through a QED perturbative expansion of the QCD+QED path integral. We compare results and statistical errors from both methods.

34th annual International Symposium on Lattice Field Theory

24-30 July 2016

University of Southampton, UK

\footnotetext{
* Speaker

†speaker.
} 


\section{Introduction}

In recent years the computation of many quantities relevant to Standard Model phenomenology in Lattice QCD has reached a precision of $\lesssim 1 \%$ [1]. However, such calculations are usually done in the isospin symmetric limit $m_{u}=m_{d}$ and without including QED effects, i.e. considering quarks as being electrically neutral. Both effects can be of the order of 1\% [2] and need to be included when aiming for calculations at this level of precision.

The treatment of electromagnetic effects in lattice calculations requires the inclusion of QED in the path integral. The expectation value of a quantity $O$ is then given in the Euclidean path integral formalism by

$$
\langle O\rangle=\frac{1}{Z} \int \mathscr{D}[U] \mathscr{D}[A] \mathscr{D}[\Psi, \bar{\Psi}] O e^{-S_{F}[\Psi, \bar{\Psi}, A, U]} e^{-S_{\gamma}[A]} e^{-S_{G}[U]}
$$

with photon fields $A$, the $S U(3)$ gauge fields $U$ and the quark fields $\Psi$ and $\bar{\Psi}$. The fermionic action $S_{F}[\Psi, \bar{\Psi}, A, U]$ now also includes couplings of quarks to photons. We follow two approaches to include QED in our lattice calculations. One is a non-perturbative treatment of QED, where $U(1)$ photon fields are generated stochastically. We generate the $U(1)$ photon fields independently of $S U(3)$ gauge fields. In this electro-quenched approximation the sea quarks are electrically neutral. The $U(1)$ photon fields are then multiplied with the $S U(3)$ gauge links [3-11]

$$
U_{\mu}(x) \rightarrow e^{i e A_{\mu}(x)} U_{\mu}(x) .
$$

In the following we will refer to this method as the stochastic method.

On the other hand QED can also be treated perturbatively by expanding the path integral (1.1) as a series in the fine structure constant $\alpha=e^{2} / 4 \pi[12]$

$$
\langle O\rangle=\langle O\rangle_{e=0}+\frac{1}{2} e^{2} \frac{\partial^{2}}{\partial e^{2}}\langle O\rangle_{e=0}+O\left(\alpha^{2}\right) .
$$

The expectation values $\langle\cdot\rangle_{e=0}$ on the right hand side of equation (1.3) are then calculated in pure QCD. We will refer to this method as the perturbative method in the following.

Strong isospin breaking effects are taken into account by putting different values for up- and down-quark masses in the valence sector. We note that a more comprehensive method for the treatment of strong isospin breaking is to expand the path integral in the isospin symmetric quark mass as proposed in [13].

A more detailed description of the stochastic and the perturbative method is given in sections 2 and 3. In sections 4 and 5 we will show results for the QED correction to meson masses and the hadronic vacuum polarization, respectively and compare results and statistical errors from both approaches.

For this exploratory study we use a $64 \times 24^{3}$ lattice with an inverse lattice spacing of $a^{-1}=$ $1.78 \mathrm{GeV}$ and $N_{f}=2+1$ dynamical flavors of Domain Wall Fermions [14]. The pion mass (without QED) is $\approx 340 \mathrm{MeV}$. We use different masses for the valence up and down quarks, such that their difference approximately corresponds to the physical mass difference [4] and we use the physical value for the valence strange quark mass [15]. We use the same bare-quark masses when caluclating with or without QED. 


\section{Stochastic Method}

In the electro-quenched approximation, $U(1)$ photon fields are generated independently of $S U$ (3) gauge fields. We define the lattice $U(1)$ vector potential at mid-links, and denote this as $A_{\mu}(x) \equiv A(x+a \hat{\mu} / 2)$. We define the non-compact photon action as:

$$
S_{\gamma}\left[A_{\mu}\right]=\frac{a^{4}}{4} \sum_{x} \sum_{\mu, v}\left(\partial_{\mu} A_{v}(x)-\partial_{v} A_{\mu}(x)\right)^{2},
$$

where $\partial_{\mu} f(x)=a^{-1}[f(x+a \hat{\mu})-f(x)]$ is the forward derivative. Using integration by parts, this can be written as

$$
S_{\gamma}\left[A_{\mu}\right]=\frac{a^{4}}{2} \sum_{x} \sum_{\mu, v} A_{\mu}(x)\left(\partial_{\mu}^{*} \partial_{v}-\delta_{\mu v} \partial^{2}\right) A_{v}(x) .
$$

where $\partial_{\mu}^{*} f(x)=a^{-1}[f(x)-f(x-a \hat{\mu})]$ is the backward derivative and $\partial^{2} \equiv \sum_{\mu} \partial_{\mu}^{*} \partial_{\mu}$. This action is invariant under constant shift transformations $A_{\mu}(x) \rightarrow A_{\mu}(x)+c_{\mu}$ and gauge transformations $A_{\mu}(x) \rightarrow A_{\mu}(x)-\partial_{\mu} f(x)$.

The shift symmetry can be eliminated by removing the zero-mode of the photon field. We choose to remove the zero-mode using the $\mathrm{QED}_{L}$ formulation [16], in which the spatial zero-mode is removed on each time slice:

$$
a^{3} \sum_{\vec{x}} A_{\mu}\left(x_{0}, \vec{x}\right)=0 \text { for all } \mu, x_{0} .
$$

This condition is the most minimal subtraction that conserves the reflection positivity of the theory with periodic boundary conditions [5]. The Feynman gauge can be imposed by adding a term to the action:

$$
\begin{aligned}
S_{\gamma, \text { Feyn. }}\left[A_{\mu}\right] & =S_{\gamma}\left[A_{\mu}\right]+\frac{1}{2} \sum_{x}\left(\sum_{\mu} \partial_{\mu} A_{\mu}(x)\right)^{2} \\
& =-\frac{a^{4}}{2} \sum_{x} \sum_{\mu} A_{\mu}(x) \partial^{2} A_{\mu}(x) .
\end{aligned}
$$

In momentum space, this action becomes

$$
S_{\gamma, \text { Feyn. }}\left[A_{\mu}\right]=\frac{1}{2 N} \sum_{k, \vec{k} \neq 0} \hat{k}^{2} \sum_{\mu}\left|\tilde{A}_{\mu}(k)\right|^{2},
$$

where $\hat{k}_{\mu}=\frac{2}{a} \sin \left(a k_{\mu} / 2\right)$ and $N$ is the number of lattice sites. All elements of the photon field $\tilde{A}_{\mu}(k)$ are therefore independent, and a momentum-space QED gauge configuration can be generated by drawing each value $\tilde{A}_{\mu}(k)$ from a Gaussian distribution with variance $2 N / \hat{k}^{2}$.

In this work, we use QED gauge configurations in both Feynman and Coulomb gauges. The Coulomb gauge fixing condition $\sum_{j} \partial_{j}^{*} A_{j}(x)=0$, where the index $j$ runs over the spatial directions, defines an operator which projects a field configuration into Coulomb gauge [5]:

$$
\begin{aligned}
A_{\mu} & \rightarrow A_{\mu}^{\prime}=\left(P_{C}\right)_{\mu v} A_{v} \\
\left(P_{C}\right)_{\mu v} & =\delta_{\mu v}-|\overrightarrow{\hat{k}}|^{-2} \hat{k}_{\mu}(0, \overrightarrow{\hat{k}})_{v} .
\end{aligned}
$$


After generating QED gauge configurations in momentum space, these are transformed into position space using an inverse Fast Fourier Transform. Since the photon field is a real vector field, the imaginary part of $A_{\mu}(x)$ is set to zero after the Fourier transform.

Once position-space photon field configurations are generated, these are multiplied with the $S U(3)$ gauge links as in (1.2) and the calculation of hadronic observables proceeds as in the case without QED. This method allows the calculations of QED corrections up to all orders within the electro-quenched approximation.

We remove $\mathscr{O}(e)$ noise by averaging expectation values evaluated with electromagnetic couplings $+e$ and $-e$, thereby restoring exact charge-conjugation symmetry on each configuration [6]. We find that this process greatly improves the signal-to-noise ratio, reducing statistical error by an order of magnitude in some cases.

To calculate electromagnetic corrections, we compute correlators both with and without QED. The stochastic method therefore requires three inversions per quark flavour per source position; for electromagnetic couplings $0,+e,-e$.

\section{Perturbative Method}

In a perturbative expansion of the path integral (1.1) in the fine structure constant $\alpha$ one obtains at lowest order diagrams with either two insertions of the conserved vector current or one insertion of the tadpole operator [12]. This results in three different types of connected diagrams (photon exchange, self energy and tadpole) which are shown in figure 1. Currently, we neglect quark-disconnected diagrams, which corresponds to quenched QED, i.e. neglecting electromagnetic effects for the sea quarks.
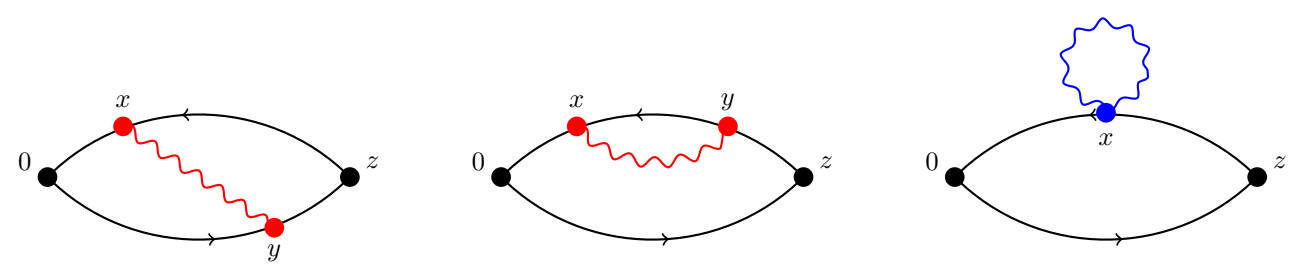

Figure 1: The three diagrams for the leading order QED corrections to mesonic two-point functions. Red and blue vertices represent insertions of the conserved vector current and the tadpole operator, respectively. From left to right the diagrams are photon exchange, self energy and tadpole.

To give an example, the photon exchange diagram for a charged kaon is given by a correlation function of the form

$$
C\left(z_{0}\right)=\sum_{\vec{z}} \sum_{x, y} \operatorname{Tr}\left[S^{s}(z, x) \Gamma_{v}^{c} S^{s}(x, 0) \gamma_{5} S^{u}(0, y) \Gamma_{\mu}^{c} S^{u}(y, z) \gamma_{5}\right] \Delta_{\mu v}(x-y),
$$

where $S^{f}(x, y)$ denotes a quark propagator from $x$ to $y$ for a quark of flavor $f, \Delta_{\mu v}(x-y)$ is the photon propagator from $x$ to $y$ and $\Gamma_{\mu}^{c}$ stands for an insertion of the conserved vector current. In Feynman gauge, the photon propagator is given by

$$
\Delta_{\mu v}(x-y)=\delta_{\mu v} \frac{1}{V} \sum_{k, \vec{k} \neq 0} \frac{e^{i k \cdot(x-y)}}{\hat{k}^{2}},
$$


with the volume of the lattice $V=L^{3} \cdot T$. In equation (3.2) we have subtracted all spatial zero modes $\vec{k}=0$ of the photon propagator, which corresponds to the formulation of $\mathrm{QED}_{L}$ [5].

To compute correlation functions such as (3.1), we rewrite the photon propagator as

$$
\Delta_{\mu v}(x-y)=\sum_{u} \Delta_{\mu v}(x-u)\left\langle\eta_{i}(u) \eta_{i}^{\dagger}(y)\right\rangle_{\eta}=\left\langle\tilde{\Delta}_{\mu v}(x) \eta^{\dagger}(y)\right\rangle_{\eta}
$$

with stochastic sources $\eta$ that have the property

$$
\left\langle\eta_{i}(u) \eta_{i}^{\dagger}(y)\right\rangle_{\eta}=\delta_{u, y} .
$$

In this work, we use $\mathbb{Z}_{2}$ sources, where we randomly pick the entries from $\left\{\frac{1}{\sqrt{2}}( \pm 1 \pm i)\right\}$. The photon propagator $\tilde{\Delta}_{\mu v}(x)$ can be calculated using a Fast Fourier Transform. We then calculate sequential quark propagators with insertions of the conserved vector current and either $\tilde{\Delta}_{\mu v}(x)$ or $\eta^{\dagger}(y)$, such that we can construct the diagrams shown in figure 1. In total this requires 17 inversions per valence quark and source position for Feynman gauge photon propagators, where only diagonal terms $\mu=v$ contribute (cf. equation (3.2)). For other gauges, e.g. Coulomb gauge, where also off-diagonal terms $\mu \neq v$ are non-zero, more inversions are required.

We use one stochastic $\mathbb{Z}_{2}$ source $\eta$ for the photon insertion per QCD gauge configuration and source position.

\section{QED correction to meson masses}

In the following we will determine the QED correction to the pion and kaon masses. Although our main interest is in calculating the QED correction to the hadronic vacuum polarization contribution to the anomalous magnetic moment of the muon, the QED correction to the meson masses can serve as a valuable cross check of our calculation and as a starting point in comparing the stochastic and the perturbative approaches.

An independent calculation of the QED correction to meson masses on the same gauge ensemble has been previously done in [7] using a stochastic approach. Those results can be used as a cross check for our implementation.

\subsection{Results obtained using the Stochastic Method}

The mass of a meson determines the leading exponential decay of the two-point function:

$$
\begin{aligned}
C(t) & =A e^{-t m}+A e^{-(T-t) m}+\ldots \\
& =2 A e^{-(T / 2) m} \cosh ((t-T / 2) m)+\ldots
\end{aligned}
$$

where $T$ is the time extent of the lattice with periodic boundary conditions. The dots represent the contributions of excited states. The mass can therefore be extracted by fitting a function of the form (4.2) to the two-point function. The QED mass correction is then defined as the correlated difference of the masses with and without QED:

$$
\delta m=m_{\mathrm{QED}}-m_{0} .
$$


A suitable fit range can be determined from the plateau region of the effective mass, defined by

$$
\frac{C(t)}{C(t+1)}=\frac{\cosh \left((t-T / 2) m_{\mathrm{eff}}\right)}{\cosh \left((t+1-T / 2) m_{\mathrm{eff}}\right)} .
$$

Figure 2 shows the mass and QED mass correction determined from a fit to the charged kaon correlator using the stochastic method, along with the corresponding effective mass. These plots use data from 87 configurations, with $16 \mathbb{Z}_{2}$ wall sources per configuration, and Coulomb-gauge $U(1)$ configurations.
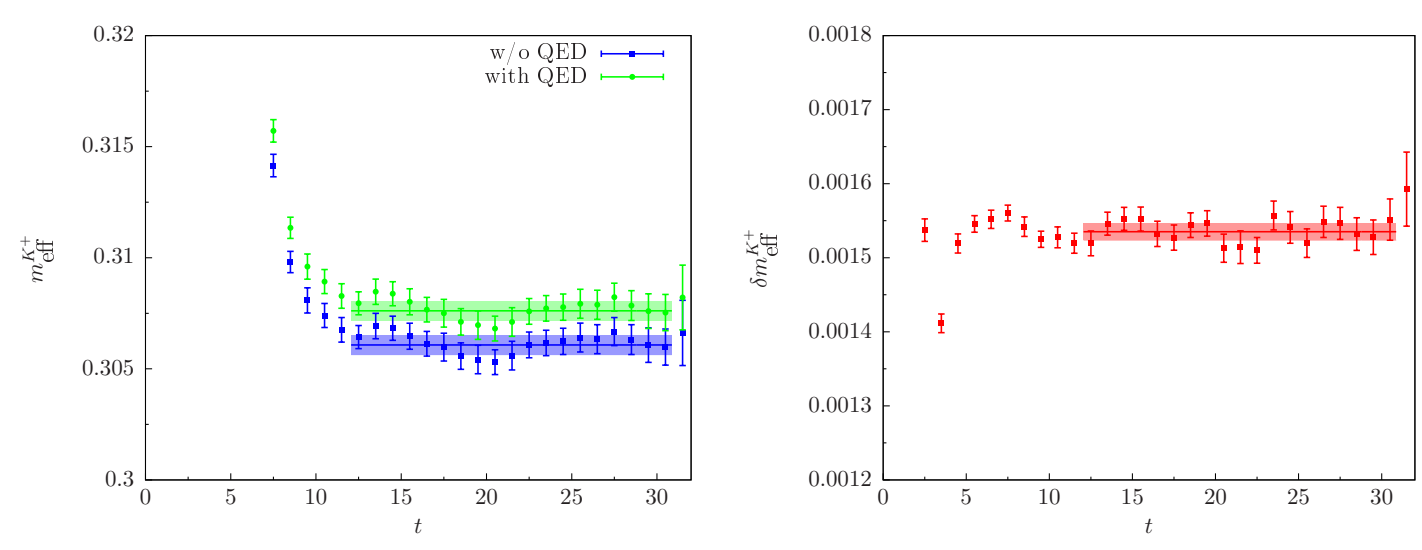

Figure 2: Effective mass of the charged kaon, along with the mass from a fit to the correlator, from the stochastic method in Coulomb gauge. The left plot shows the charged kaon mass with and without QED, and the right plot shows the QED mass correction $\delta m$.

We have calculated the QED mass correction using the stochastic method on the same statistics with Feynman-gauge $U(1)$ configurations. Figure 3 shows the correlated difference of the charged kaon effective mass QED correction evaluated in the Feynman and Coulomb gauges. At early times, one can observe a disagreement between the two gauges that can be explained in the following way. In this region the effective mass is contaminated by excited states and in particular depends on their creation amplitude, which is a gauge-dependent number. At later times, we see an expected agreement between the different gauges.

In table 1 we compare our values for the pion squared mass splitting $\Delta m^{2}=m_{\mathrm{QED}}^{2}-m_{0}^{2}$ with those from [7]. Both analyses use a stochastic method with Feynman-gauge $U(1)$ configurations, the same $S U$ (3) gauge ensemble and the same fit range. We find agreement between our work and the independent study at $1 \sigma$.

\begin{tabular}{|c|c|c|c|}
\hline$q_{1}$ & $q_{2}$ & $\Delta m^{2}$ from this work & $\Delta m^{2}$ from [7] \\
\hline \hline $2 / 3$ & $2 / 3$ & $(5.465 \pm 0.035) \times 10^{-4}$ & $(5.406 \pm 0.064) \times 10^{-4}$ \\
$2 / 3$ & $-1 / 3$ & $(7.677 \pm 0.052) \times 10^{-4}$ & $(7.654 \pm 0.056) \times 10^{-4}$ \\
$-1 / 3$ & $-1 / 3$ & $(1.341 \pm 0.009) \times 10^{-4}$ & $(1.326 \pm 0.016) \times 10^{-4}$ \\
\hline
\end{tabular}

Table 1: Comparison of pion squared mass splittings from this study, using the stochastic method in Feynman gauge, with those published in [7]. Both quark masses are equal to the unitary light mass. Quark charges are in units of the electron charge $e$, and mass splittings are in lattice units. 


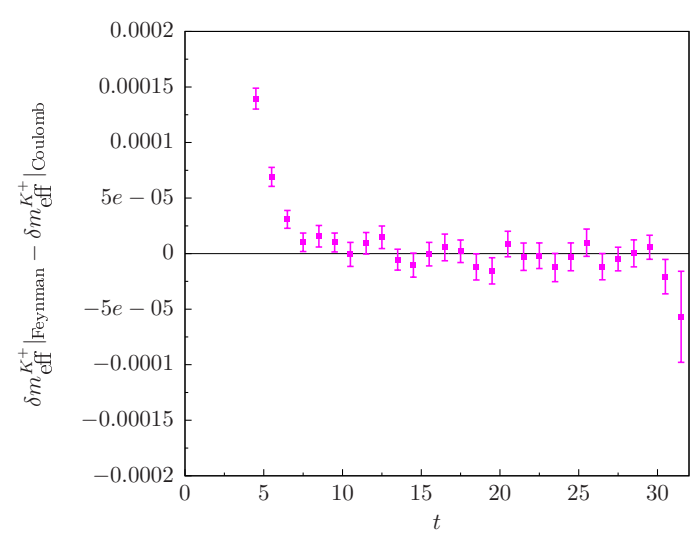

Figure 3: Correlated difference of the charged kaon effective mass QED correction evaluated in the Feynman and Coulomb gauges, using the stochastic method.

Table 2 contains the QED corrections to pion and kaon masses from the stochastic method in Feynman gauge alongside results from the perturbative method. These results have not been corrected for finite volume effects. An analytic formula to correct for finite volume effects in the meson masses is given in [5].

\subsection{Results obtained using the Perturbative Method}

The exponential decay of the two-point function including QED is determined by the mass $m_{0}$ without QED plus the leading order QED correction $\delta m$

$$
C(t)=C_{0}(t)+C_{O(\alpha)}(t)+O\left(\alpha^{2}\right)=A e^{-\left(m_{0}+\delta m\right) \cdot t} .
$$

Expanding the exponent in (4.5) one finds that the QED correction to the meson mass can be estimated from the slope of the ratio of the QED correction $C_{O(\alpha)}(t)$ to the two-point function and the two-point function $C_{0}(t)$ without QED [12]

$$
\delta m=-\frac{\partial}{\partial t} \frac{C_{O(\alpha)}(t)}{C_{0}(t)} .
$$

Indeed we find a linear behavior in our data for $C_{O(\alpha)}(t) / C_{0}(t)$ as can be seen in figure 4 . This plot shows the correlation functions of the three different types of QED correction divided by the two-point function without QED for a charged kaon. We have fitted a linear function to the data to obtain the slope, which can be related to the QED correction by (4.6).

In table 2 results for the QED correction to meson masses from the stochastic and the perturbative method are listed. The values are given in lattice units and have not been corrected for finite volume effects. Besides the QED corrections to the masses of charged and neutral pion as well as charged and neutral kaon, we quote the pion mass splitting $M_{\pi^{+}}-M_{\pi^{0}}$, which is given only by the photon exchange diagram, the contributions from the other diagrams cancel [12]. Note, that we do not include the disconnected diagram for the neutral pion.

In addition, we have calculated the QED corrections to the meson masses with the perturbative method using the Coulomb gauge on a subset of the statistics and find agreement between the results in both gauges. 


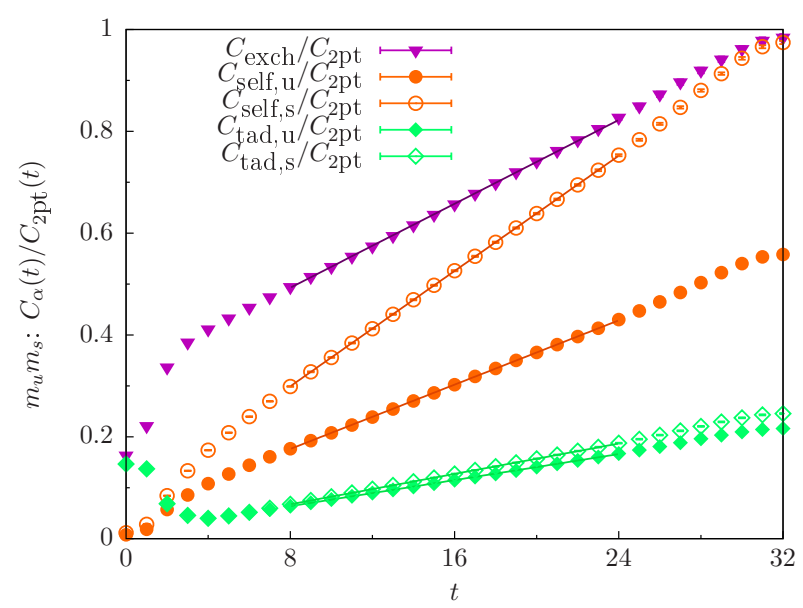

Figure 4: The QED correction to the mass of a charged kaon from the perturbative method. Orange circles and violet triangles are the contributions from the self energy and photon exchange diagram, respectively. The green diamonds are the contribution from the tadpole diagram. Where open and closed symbols are used, the open symbols refer to the photon being attached to the s quark, the closed symbols to the photon being attached to the u quark. Charge factors are not included in the data shown here.

\begin{tabular}{|c|c|c|}
\hline quantity & stochastic & perturbative \\
\hline \hline$a M_{\pi^{+}}^{\gamma}$ & $(1.968 \pm 0.013) \times 10^{-3}$ & $(1.963 \pm 0.016) \times 10^{-3}$ \\
$a M_{\pi^{0}}^{\gamma}$ & $(0.885 \pm 0.007) \times 10^{-3}$ & $(0.877 \pm 0.016) \times 10^{-3}$ \\
$a\left(M_{\pi^{+}}-M_{\pi^{0}}\right)$ & $(1.148 \pm 0.013) \times 10^{-3}$ & $(1.115 \pm 0.019) \times 10^{-3}$ \\
\hline$a M_{K^{+}}^{\gamma}$ & $(1.532 \pm 0.011) \times 10^{-3}$ & $(1.513 \pm 0.013) \times 10^{-3}$ \\
$a M_{K^{0}}^{\gamma}$ & $(0.310 \pm 0.002) \times 10^{-3}$ & $(0.309 \pm 0.004) \times 10^{-3}$ \\
\hline
\end{tabular}

Table 2: Results for the QED corrections to the meson masses in lattice units from the stochastic and perturbative method without finite volume correction.

\subsection{Comparison of both methods}

To compare the results from the stochastic and the perturbative methods, we determine the QED correction to the effective mass. For the stochastic data, we simply calculate the effective mass once for the two-point function including QED and once for the two-point function without QED and calculate their difference

$$
\delta m_{\mathrm{eff}}(t)=m_{\mathrm{eff}}^{\mathrm{QED}}(t)-m_{\mathrm{eff}}^{0}(t) .
$$

For the perturbative method we make use of equation (4.6) and determine the QED correction to the effective mass as

$$
\delta m_{\mathrm{eff}}(t)=\frac{C_{O(\alpha)}(t)}{C_{0}(t)}-\frac{C_{O(\alpha)}(t+1)}{C_{0}(t+1)} .
$$

The left-hand side of figure 5 shows the QED correction to the effective mass of a charged kaon obtained in both methods using the Feynman gauge for the photon fields. Blue circles show the results from the stochastic method, red squares the results from the perturbative method. The plot on the right-hand side of figure 5 side shows the correlated difference of both datasets. Both 
datasets have been obtained on the same 87 QCD gauge configurations using 16 source positions for the stochastic and 4 source positions for the perturbative method. For the 4 source positions both datasets have in common we have used the same $\mathbb{Z}_{2}$ wall sources for the quark propagators. We find good agreement between the data from both methods. Note, that one expects both approaches to differ at $O\left(\alpha^{2}\right)$, however, our data suggests, that these effects are smaller than the statistical precision.
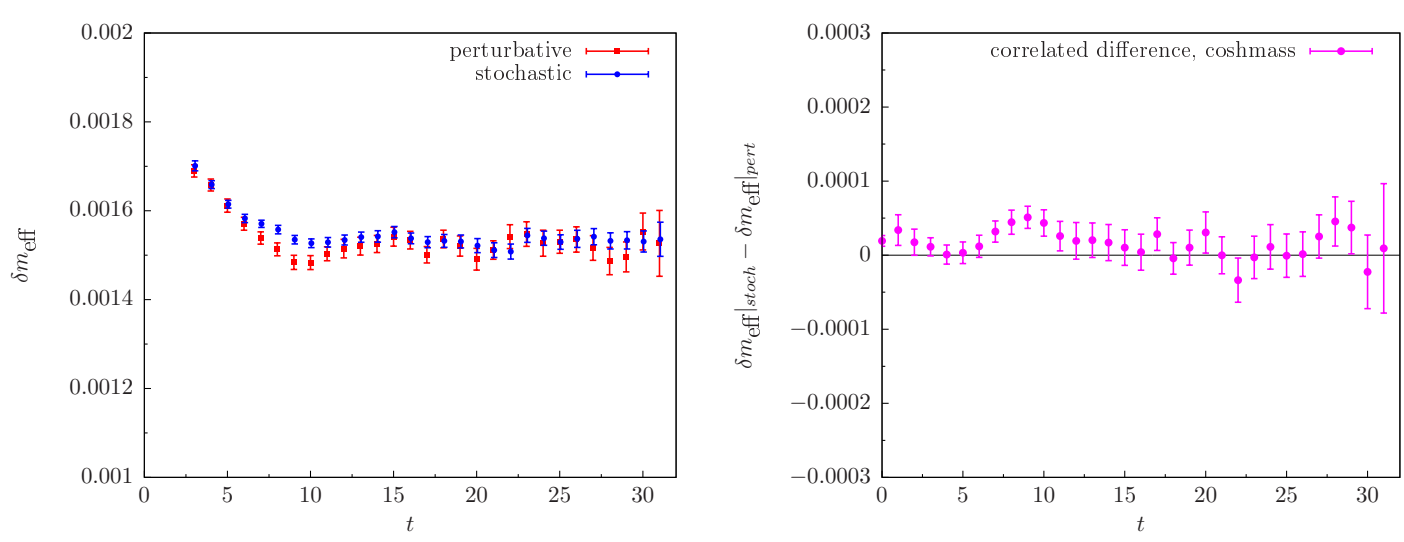

Figure 5: The QED correction to the effective mass for a charged kaon is shown on the left. Blue circles show results from the stochsatic method, red squares results from the perturbative method. The plot on the right-hand side shows the correlated difference of both datasets.

For a comparison of the statistical precision of both methods, one has to take into account that the results have not been obtained at equal cost. For each quark flavor and source position the stochastic method requires three inversions $(q=0,+e,-e)$. In the Feynman gauge (where only diagonal terms $\Delta_{\mu \mu}$ of the photon propagator contribute), the perturbative method requires 17 inversions per quark flavor and source position to construct all the diagrams shown in figure 1. Thus, for the result shown here, the computational cost is $3 \times 16$ (\# inversions $\times \#$ source positions) for the stochastic and $17 \times 4$ for the perturbative method.

In figure 6 we compare the statistical errors of the QED correction to the effective kaon mass for both methods. The left-hand side shows the ratio of the errors from the perturbative method over the stochastic method, both scaled with the numerical cost. The horizontal black line shows "1", i.e. where both methods would be equal in statistical precision. However, we find that the error on the perturbative approach is about 1.5-2 times larger than the error on the stochastic approach for the same computational cost. This might of course be a mass-dependent finding and differ for the physical values of the quark masses.

The right-hand side of figure 6 shows a comparison of errors on the same set of statistics (using the same 4 source positions in both data sets). Note, that this is not an equal cost comparison. Here we find both methods to have the same level of precision, indicating, that most of the noise originates from the QCD average. 

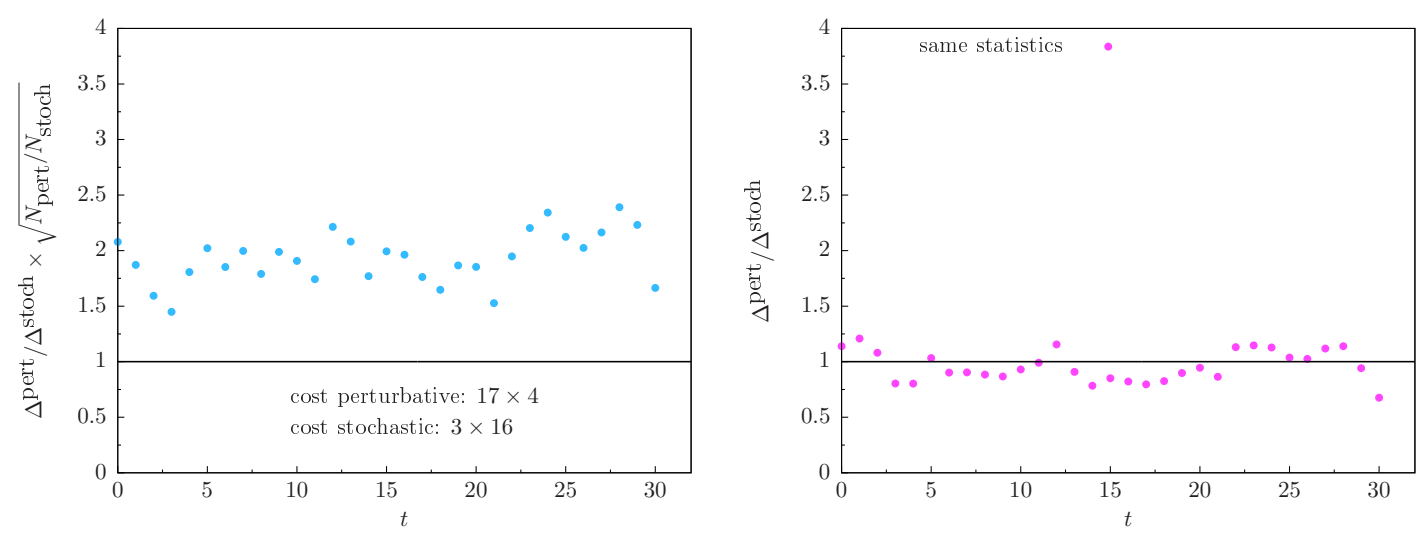

Figure 6: Comparison of the statistical error on the QED correction to the effective kaon mass. On the plot on the left the errors for both methods are scaled with their respective computational cost. The plot on the right shows a comparison of statistical errors using the same amount of statistics (4 source positions).

\section{QED correction to the hadronic vacuum polarization}

\subsection{Introduction}

The hadronic vacuum polarization (HVP) is the leading order hadronic contribution to the anomalous magnetic moment of the muon $a_{\mu}$. Since many years a deviation of about $3 \sigma$ between the experimental value for $a_{\mu}$ and the Standard Model prediction persists, leaving room for new physics. Currently the most precise prediction for the HVP is obtained from the cross section of $e^{+} e^{-} \rightarrow$ hadrons [17] with a precision of $\lesssim 1 \%$. In the past few years substantial progress has been made in calculating the HVP in Lattice QCD (see e.g. [18-22] ) aiming to be competitive with the prediction from $e^{+} e^{-} \rightarrow$ hadrons.

In this work we report a first attempt to estimate the QED corrections to the hadronic vacuum polarization. We calculate the HVP using a conserved vector current at the sink and a local current at the source

$$
C_{\mu v}(x)=Z_{V} q_{f}^{2}\left\langle V_{\mu}^{c}(x) V_{v}^{\ell}(0)\right\rangle .
$$

Since one of the currents is non-conserved, we have to renormalize with the appropriate factor $Z_{V}$, which we take from a determination of the pion vector three-point function [23]. The HVP tensor is constructed as [22]

$$
\Pi_{\mu v}(Q)=\sum_{x} e^{-i Q \cdot x} C_{\mu v}(x)-\sum_{x} C_{\mu v}(x)
$$

with a subtraction of the zero-mode $\sum_{x} C_{\mu v}(x)[22,24]$. For the HVP form factor we only consider contributions from (5.2) with vanishing spatial momentum $Q_{i}=0$ and estimate $\Pi\left(\hat{Q}^{2}\right)$ as

$$
\Pi\left(\hat{Q}^{2}\right)=\frac{1}{3} \sum_{j} \frac{\Pi_{j j}(Q)}{\hat{Q}^{2}}
$$

with $j=1,2,3$. From the HVP form factor the hadronic contribution to $a_{\mu}$ can be calculated as [25]

$$
a_{\mu}^{\mathrm{HVP}}=\left(\frac{\alpha}{\pi}\right)^{2} \int_{0}^{\infty} \mathrm{d} Q^{2} K\left(Q^{2}\right)\left[\Pi\left(Q^{2}\right)-\Pi(0)\right] .
$$




\subsection{Results}

For the QED correction to the HVP calculated from the perturbative method, we have to consider two additional types of diagrams (cf figure 7) further to the diagrams in figure 1. These diagrams originate from a perturbative expansion in $\alpha$ of the conserved vector current at the sink. These two diagrams do not require any additional inversions.
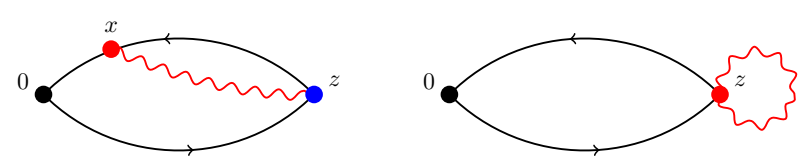

Figure 7: The additional diagrams for the QED correction to the HVP that occur when a conserved current is used at the sink. Red and blue vertices represent insertions of the conserved vector current and the tadpole operator, respectively.

Figures 8 and 9 show results for the HVP form factor without QED (plots on the left) and the QED correction (plots on the right) for up and strange quarks, respectively. On the right-hand side plots, we show data obtaind from the stochastic method (blue circles) and the perturbative method (red squares). We find agreement between the results for the QED correction to the HVP from both methods. To obtain this consistency it is crucial to include the contact terms in figure 7 in the perturbative approach.
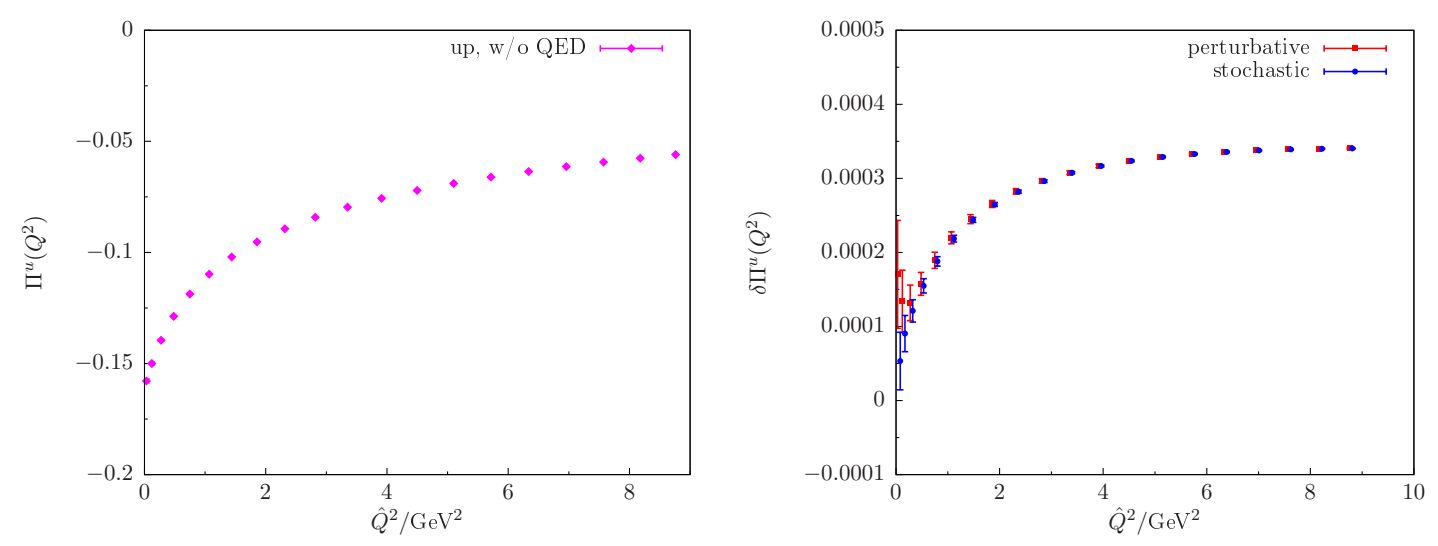

Figure 8: The up quark hadronic vacuum polarization form factor $\Pi\left(Q^{2}\right)$ without $Q E D$ is shown on the left. The plot on the right shows the QED correction to the HVP form factor for the u quark. Blue circles show data obtained using the stochastic method, red squares show data from the perturbative method.

To get a first glimpse of the size of the QED correction to the anomalous magnetic moment of the muon we use [24]

$$
\hat{\Pi}\left(\hat{Q}^{2}\right)=\Pi\left(\hat{Q}^{2}\right)-\Pi(0)=2 \sum_{z} C_{j j}(z)\left[\frac{z_{0}^{2}}{2}-\frac{1-\cos \left(Q z_{0}\right)}{Q^{2}}\right]
$$

to renormalize the HVP form factor. We fill the momenta $Q^{2}$ with non-lattice momenta using a sine cardinal interpolation [22] and integrate naivly using a trapezoidal rule to obtain $a_{\mu}$ according to equation (5.4). However, in this way we are, at the current level of statistics, not able to resolve the 

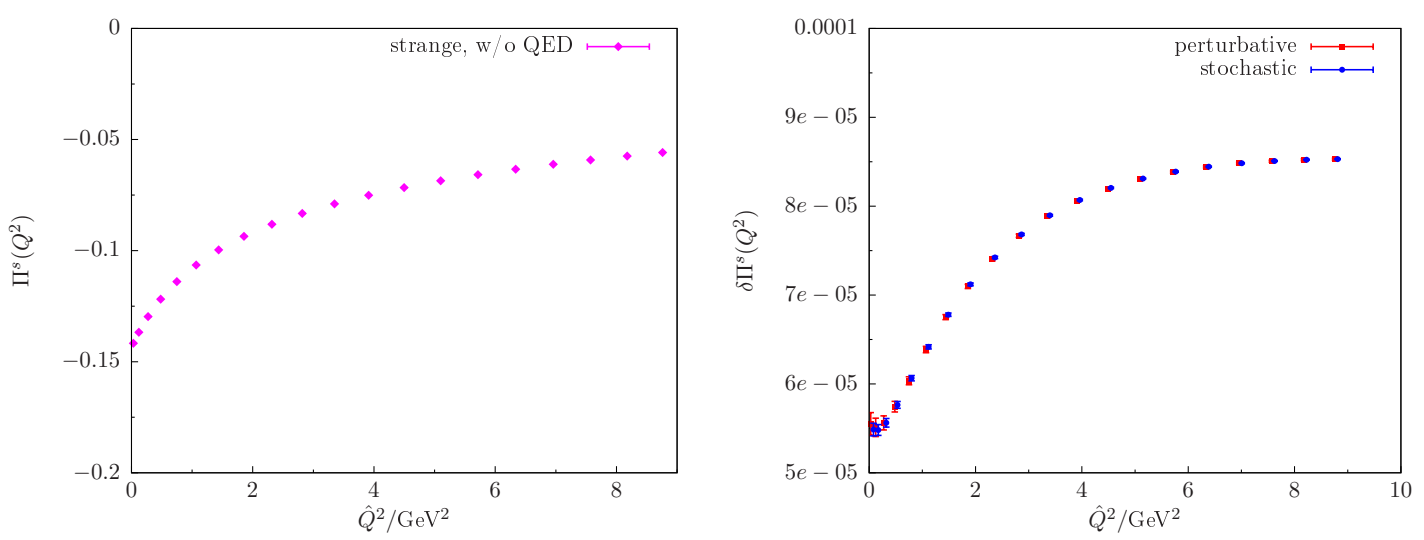

Figure 9: The same as figure 8 for the strange quark.

QED correction to the renormalized HVP $\hat{\Pi}\left(\hat{Q}^{2}\right)$ in the low- $Q^{2}$ region which is most relevant for $a_{\mu}$. Thus, we obtain the QED corrections to $a_{\mu}$, which are consistent with zero within the statistical errors. Nevertheless, this still allows us to quote an upper limit for the QED corrections, which we find to be $\lesssim 1 \%$ for the up quark, and even smaller for down and strange, where the QED correction is suppressed by a factor 4 compared to the up, due to the charge factors from the internal coupling of photons.

In addition, one has to consider the QED correction to the multiplicative renormalization $Z_{V}$ for the local vector current in the HVP

$$
Z_{V}=Z_{V}^{0}+\alpha Z_{V}^{1}
$$

which yields at $O(\alpha)$ a further QED correction to the HVP of the form $Z_{V}^{1} \Pi^{0}\left(\hat{Q}^{2}\right)$ with $\Pi^{0}\left(\hat{Q}^{2}\right)$ the HVP without QED. We aim to include this correction to $Z_{V}$ in our future work.

\subsection{Comparison of statistical error}

In figure 10 we show the ratio of the statistical errors of the perturbative method over the stochastic method, scaled with the computational cost. The horizontal black line shows "1", i.e. where both methods would be equal in statistical precision. We find the stochastic method to give an $\approx 2$ times smaller statistical error for the HVP form factor and $2-2.5$ times for the renormalized HVP for the same numerical cost (i.e. the same number of inversions). We find this to be independent of the quark masses within the range studied here.

\section{Summary and Outlook}

We have calculated the QED corrections to meson masses and the hadronic vacuum polarization using two different approaches - a stochastic approach using $U(1)$ gauge configurations for the photon fields, and a perturbative approach by expanding the path integral in the electromagnetic coupling $\alpha$. We find agreement between the results from both methods, and with a previous independent determination [7] of the QED corrections to the meson masses on the same gauge ensemble using a stochastic approach. 

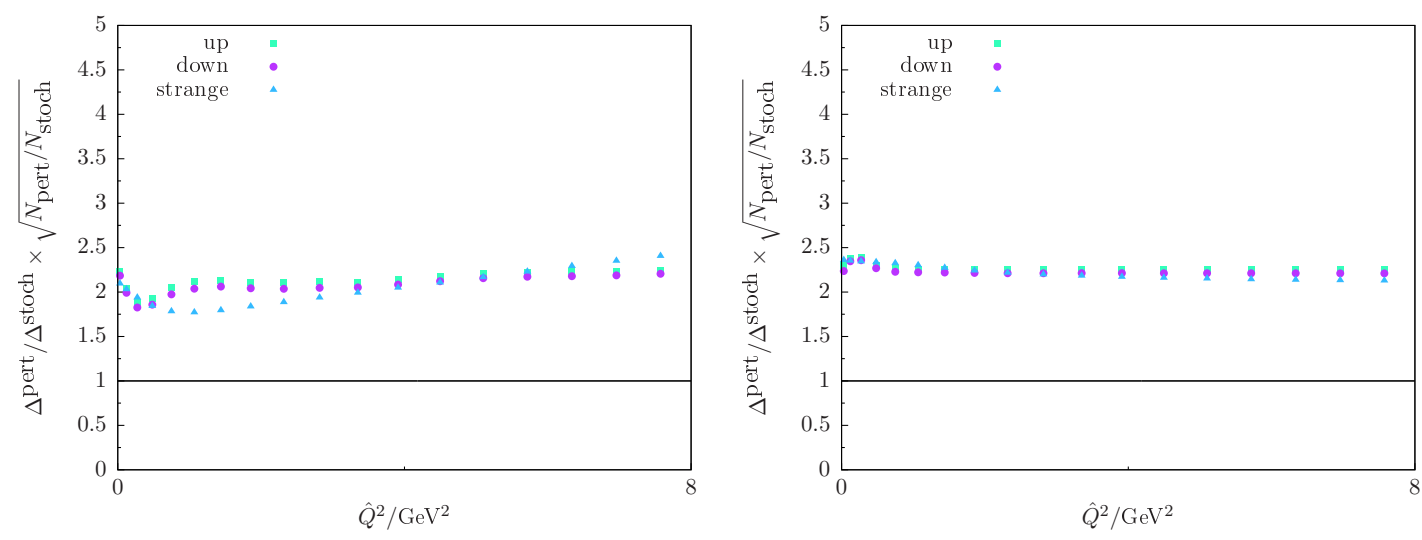

Figure 10: Comparison of the statistical errors on the QED correction to the HVP from both methods for the HVP form factor (left) and the renormalized HVP (right). The plots show the ratio of the errors from the perturbative method over the stochastic method, both scaled with the numerical cost.

Comparing the statistical errors on the QED corrections from both approaches for the meson masses and the HVP, we find the stochastic approach to yield a factor of $\approx 2$ smaller errors for the same numerical cost, i.e. the number of inversions.

We find the QED correction to the anomalous magnetic moment to be consistent with zero within the statistical errors, but at most of the order of $1 \%$. Using a more sophisticated way to determine the additive renormalization $\Pi(0)$, e.g. using Padé approximants, we might be able to resolve $\hat{\Pi}\left(\hat{Q}^{2}\right)$ in the low- $Q^{2}$ region. In addition, we are currently increasing statistics for the QED correction to the HVP, and investigating the use of all-mode-averaging [26].

We plan to estimate the QED correction to the multiplicative renormalization for the local vector current $Z_{V}$, which results in a further QED correction of the form $Z_{V}^{1} \Pi^{0}\left(\hat{Q}^{2}\right)$. A more complete picture of the QED correction to the HVP will also require the inclusion of certain quarkdisconnected diagrams, like the one shown in figure 11, which is not $S U(3)$ flavor suppressed, in contrast to its counterpart without $\mathrm{QED}$, i.e. without a photon coupling the two quark loops.

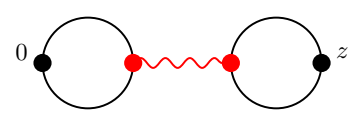

Figure 11: Disconnected diagram for the QED correction to the HVP.

Another important issue that we will address in the future is a study of finite volume effects for the QED correction to the HVP. Finite volume corrections with photons in a finite box are expected to be significant, and it is therefore important to include them to obtain a precise result.

\section{Acknowledgments}

This work has received financial support from the EPSRC Centre for Doctoral Training in Next Generation Computational Modelling grant EP/L015382/1. This work has received financal support from the STFC Grant ST/L000296/1. The research leading to these results has received 
funding from the European Research Council under the European Union's Seventh Framework Programme (FP7/2007-2013) / ERC Grant agreement 279757. P.A.B. and A.P. are supported in part by UK STFC grant ST/L000458/1. This work used the DiRAC Blue Gene Q Shared Petaflop system at the University of Edinburgh, operated by the Edinburgh Parallel Computing Centre on behalf of the STFC DiRAC HPC Facility (www.dirac.ac.uk). This equipment was funded by BIS National E-infrastructure capital grant ST/K000411/1, STFC capital grant ST/H008845/1, and STFC DiRAC Operations grants ST/K005804/1 and ST/K005790/1. DiRAC is part of the National EInfrastructure.

\section{References}

[1] S. Aoki et al., arXiv:1607.00299 (2016)

[2] A. Portelli, PoS LATTICE2014, 013 (2015), [arXiv:1505.07057]

[3] A. Duncan, E. Eichten, and H. Thacker, Phys. Rev. Lett. 76, 3894 (1996), [arXiv:hep-lat/9602005]

[4] Z. Fodor et al., Phys. Rev. Lett. 117, 082001 (2016), [arXiv:1604.07112]

[5] S. Borsanyi et al., Science 347, 1452 (2014), [arXiv:1406.4088]

[6] T. Blum et al., Phys. Rev. D76, 114508 (2007), [arXiv:0708.0484]

[7] T. Blum et al., Phys. Rev. D82, 094508 (2010), [arXiv:1006.1311]

[8] S. Borsanyi et al., Phys. Rev. Lett. 111, 252001 (2013), [arXiv:1306.2287]

[9] R. Horsley et al., JHEP 04, 093 (2016), [arXiv:1509.00799]

[10] R. Horsley et al., J. Phys. G43, 10LT02 (2016), [arXiv:1508.06401]

[11] S. Basak et al., PoS LATTICE2015, 259 (2016), [arXiv:1606.01228]

[12] G. M. de Divitiis et al., Phys. Rev. D87, 114505 (2013), [arXiv:1303.4896]

[13] G. M. de Divitiis et al., JHEP 04, 124 (2012), [arXiv:1110.6294]

[14] C. Allton, Phys. Rev. D78, 114509 (2008), [arXiv:0804.0473]

[15] T. Blum et al., Phys. Rev. D93, 074505 (2016), [arXiv:1411.7017]

[16] S. Uno and M. Hayakawa, Prog. Theor. Phys. 120, 413-441 (2008), [arXiv:0804.2044]

[17] M. Davier et al., Eur. Phys. J. C71 1515 (2011), [arXiv:1010.4180]

[18] P. Boyle et al., Phys. Rev. D85, 074504 (2012), [arXiv:1107.1497]

[19] M. Della Morte et al., JHEP 03, 055 (2012), [arXiv:1112.2894]

[20] F. Burger et al., JHEP 02, 099 (2014), [arXiv:1308.4327]

[21] B. Chakraborty et al., arXiv:1601.03071 (2016)

[22] T. Blum et al., JHEP 04, 063 (2016), [arXiv:1602.01767]

[23] P. Boyle et al. JHEP 06,164 (2015), [arXiv:1504.01692]

[24] D. Bernecker, and H. Meyer, Eur. Phys. J. A47 148 (2011), [arXiv:1107.4388]

[25] T. Blum, Phys. Rev. Lett. 91, 052001 (2003), [arxiv:hep-lat/0212018]

[26] T. Blum, T. Izubuchi, and E. Shintani, Phys. Rev. D88, 094503 (2013), [arXiv:1208.4349] 